\title{
Calibration of a Nonlinear DC Motor under Uncertainty Using Nonlinear Optimization Techniques
}

\author{
Hamed Keshmiri Neghab", Hamid Keshmiri Neghab2 \\ ${ }^{1}$ Control Engineering Department, Faculty of Electrical Engineering, Ferdowsi University of Mashhad, Mashhad, \\ P. O. B. 9177948974 , Iran \\ 2 Department of Theory of Electrical Engineering, Faculty of Electrical Engineering, University of West Bohemia, 30100 Pilsen, \\ Univerzitní 2732/8, Czech Republic \\ * Corresponding author, e-mail: neghab@kte.zcu.cz
}

Received: 08 April 2020, Accepted: 12 June 2020, Published online: 28 January 2021

\begin{abstract}
The use of DC motors is increasingly high and it has more parameters which should be normalized. Now the calibration of each parameters is important for each motor, because it affects in its performance and accuracy. A lot of researches are investigated in this area. In this paper demonstrated how to estimate the parameters of a Nonlinear DC Motor using different Nonlinear Optimization techniques of fitting parameters to model, that called model calibration. First, three methods for calibration of a DC motor are defined, then unknown parameters of the mathematical model with the nonlinear optimization techniques for the fitting routines and model calibration process, are identified. In addition, three optimization techniques such as Levenberg-Marquardt, Constrained Nonlinear Optimization and Gauss-Newton, are compared. The goal of this paper is to estimate nonlinear parameters of a DC motor under uncertainty with nonlinear optimization methods by using LabVIEW software as an industrial software and compare the nonlinear optimization methods based on position, velocity and current. Finally, results are illustrated and comparison between these methods based on the results are made.
\end{abstract}

Keywords

calibration, Levenberg-Marquardt, Constrained Nonlinear Optimization, Gauss-Newton Optimization, uncertainty

\section{Introduction}

A DC motor is a kind of Electrical motors that convert current electrical energy to mechanical energy. The speed of a DC motor by applying variable voltage and currents, can be controlled. In this paper, a motor is assumed not a generator. DC motors are used in many applications and it becomes a popular case of study among researchers. Nowadays, DC motors are increasingly used in industrial applications, and for performing accurately, they must be calibrated. Calibration of DC motors, both large and small motors, is an important field of study in recent years among scientists and each DC motor must be calibrated for its specific application.

A lot of techniques for improving the performance of DC motor are proposed. The problem of optimum Kalman Filter for the stochastic systems which are linear and discrete and includes multiplicative noises and sensors with random two-step delays are proposed in [1] and [2], and a method for the state space control of a DC motor are proposed in [3]. The state feedback control which is linear completed by using a feedforward control because of the relief of Coulomb friction. Chevrel et al. in [4], proposed an approach for modeling of an effective DC-motor speed controlling relying on switched quadratic regulators is proposed.

To improve the linear brushless direct current motor, in [5] a method of modified linear-quadratic optimal control is presented. Various optimization techniques are applied to solve suchlike problems [6]. Metaheuristics are general heuristic search patterns and strategies which are not specified for a particular problem [7]. An overview of optimization methods is proposed in [8]. Also, a new optimization framework is proposed by Pánek et al. in [9].

For controlling of permanent magnet DC motors some methods like extended Kalman filter and Space vector modulation are represented in [10-12].

A dual-layer optimization method is proposed in [13], first a nonlinear optimization method for finding the optimum parameters and then a metaheuristic optimization 
method is defined to estimate the best parameters. Roy and Srivastava in [14], designed a PID controller in order to control the speed of a permanent magnet DC motor. They used constrained particle swarm optimization technique. Also, another design for the permanent magnetic BLDC motor is proposed for finding the optimal magnetic density field as the main objective of this problem by finding the optimum parameters [15]. Moreover, a new stochastic optimization algorithm based on biology investigated by Sundareswaran and Bhattacharjee in [16].

Gauss-Newton Optimization is another well-known method which is investigated by many researchers [17-19]. Levenberg-Marquardt technique is used to solve non-linear least-square problems. Its application in parameter estimation is very popular among researchers [20-22]. A robust speed control of a motor is proposed by Zeb et al. in [23], by using a fuzzy logic controller. Alhanjouri [24], investigated an artificial neural network in order to control the speed of a DC motor.

This paper demonstrates how to estimate and compare the parameters of a Nonlinear DC motor using different techniques of fitting parameters to model, here called model calibration. In this study, the signals of the drive current $(i)$, motor position (shaft angle $(\theta)$ ), motor velocity (Angular velocity $(w=\dot{\theta}))$ are measured by the sensor. This paper is implemented by LabVIEW software and three nonlinear optimization methods on nonlinear parameters of a DC motor under uncertainty are used, and in order to approve which methods perform better than others the comparison is made.

Section 2 is a description of the system of a DC motor and its equations. Methods are described in Section 3, models and results are defined in Section 4 and Section 5, respectively. Section 6 is the conclusion.

\section{Description}

In this paper, the calibration of DC motor by using nonlinear optimization techniques such as constraint nonlinear optimization technique, Guass-Newton optimization and Levenberg-Marquardt fit theory are compared.

Here, first, the range of parameters and maximum and minimum ranges are specified. Also, the initial conditions for the fitting routines are defined and it will be clear that some methods are very susceptible to the initial conditions.

Then unknown parameters of the mathematical model with the different nonlinear optimization techniques for the fitting routines and model calibration process are defined. Moreover, the techniques for model calibration are chosen. Also, after choosing the technique, notice that some parameters are specific for the technique used. In addition, the parameters can be changed directly by modifying the parameters control.

When the model calibration process is done, the parameters can be accepted to transfer the optimum values as initial conditions. Also a doublet for excitation are created and all measured responses are under uncertainty.

The electric circuit of the armature and layout of the rotor demonstrated in the following Fig. 1.

DC motor variables are electric resistance $(R)$, inductance $(L)$, motor constant $\left(K_{e}\right)$, armature constant $\left(K_{t}\right)$, Moment of inertia of the rotor $(J)$, and damping ratio of the mechanical system $(B)$.

Source voltage $(V)$ is input and drive current $(i)$, motor position (shaft angle $(\theta)$ ) and motor velocity (Angular velocity $(w=\dot{\theta}))$ are outputs. The rotor and shaft are assumed to be rigid. For this study, in Table 1, the following values for the physical parameters will be considered. These values are measured before.

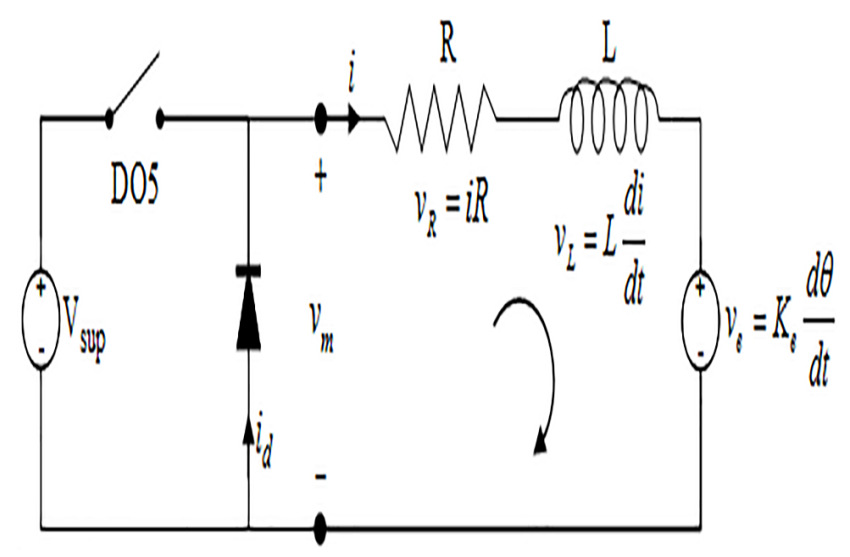

(a)

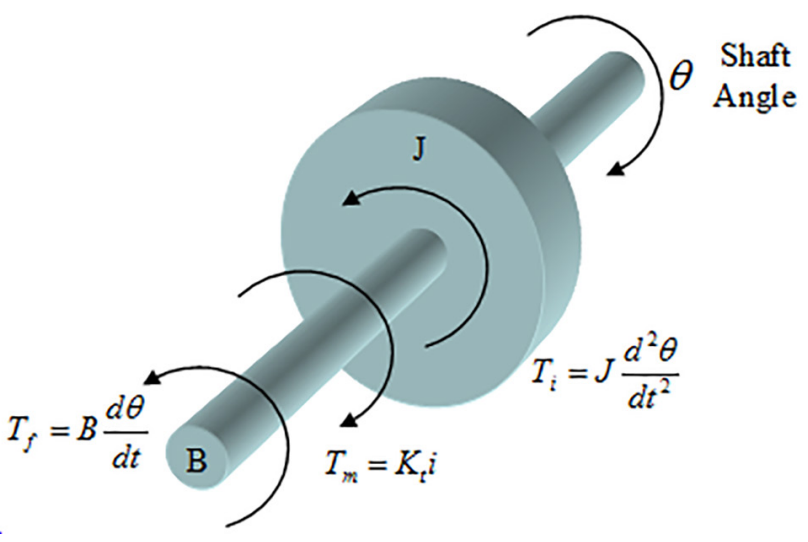

(b)

Fig. 1 The electric circuit of the armature and diagram of the rotor; (a) Electric circuit; (b) Armature diagram. 
Table 1 Real values of physical parameters

\begin{tabular}{lc}
\hline Parameters & Real value \\
\hline$R(\mathrm{Ohm})$ & 3 \\
$L($ Henry $)$ & 0.4 \\
$K_{e}(\mathrm{~N} \mathrm{~m} / \mathrm{A})$ & 0.0025 \\
$K_{t}(\mathrm{~N} \mathrm{~m} / \mathrm{A})$ & 0.0025 \\
$J\left(\mathrm{Kg} \mathrm{m}^{2} / \mathrm{s}^{2}\right)$ & 0.005 \\
$B(\mathrm{~T})$ & 0.1 \\
\hline
\end{tabular}

\subsection{System equations}

Mathematical model and the dynamic equations in statespace form are the following Eq. (1) and Eq. (2):

$T=K_{t} i$,

$e=K_{e} \dot{\theta}$

The motor torque $(T)$, is relevant to the armature current $(i)$, by a armature constant factor $\left(K_{t}\right)$. The back electromotive force $(e), \dot{\theta}$ is angular velocity and motor constant factor $\left(K_{e}\right)$ is defined.

From the Fig. 1, the below equations relying on Newton's law composed by Kirchhoff's law can be written:

$J \ddot{\theta}+b \dot{\theta}=K i$,

$L \frac{d i}{d t}+R i=V-K \dot{\theta}$

The above equations can be represented in terms of $s$ by using Laplace transformation:

$s\left(J_{s}+b\right) \theta(s)=K I(s)$,

$(L s+R) I(s)=V-K s \theta(s)$.

By removing $I(s)$ the following transfer function can be achieved:

$\frac{\theta}{V}=\frac{K}{s\left(\left(J_{S}+b\right)(L s+R)+K^{2}\right)}$.

However, during this study, the position $(\theta)$ was looked at as the output. The position can be obtained by integrating theta dot, therefore it just needed to distribute the transfer function by $s$ :

$\frac{\theta}{V}=\frac{K}{s\left(\left(J_{S}+b\right)(L s+R)+K^{2}\right)}$.

So, the dynamic equations in state-space form are as follow:

$$
\begin{aligned}
& {\left[\begin{array}{c}
\dot{\theta} \\
\ddot{\theta} \\
\dot{I}
\end{array}\right]=\left[\begin{array}{ccc}
0 & 1 & 0 \\
0 & \frac{-b}{J} & \frac{K_{t}}{J} \\
0 & \frac{-K_{e}}{L} & \frac{-R}{L}
\end{array}\right]\left[\begin{array}{l}
\theta \\
\dot{\theta} \\
I
\end{array}\right]+\left[\begin{array}{l}
0 \\
0 \\
\frac{1}{L}
\end{array}\right] V,} \\
& {\left[\begin{array}{l}
y_{1} \\
y_{2} \\
y_{3}
\end{array}\right]=\left[\begin{array}{lll}
1 & 0 & 0 \\
0 & 1 & 0 \\
0 & 0 & 1
\end{array}\right]\left[\begin{array}{l}
\theta \\
\dot{\theta} \\
I
\end{array}\right] .}
\end{aligned}
$$

\section{Methods}

In Section 3, nonlinear optimization techniques to estimate the parameters of a nonlinear equation of fitting parameters to model Eq. (3), are described. The parameters and equations of a DC motor are nonlinear and these three methods are famous in problems with nonlinear constraints or objective functions. In experimental science, some simple data analysis can be achieved by using linear techniques, however, in general, these kinds of problems are also nonlinear. Typically, a theoretical model of the system which is under discussion with changing parameters in it and an experiment model which may also have passive parameters. This makes an effort to meet a numerically best fit. In these conditions, one desires a measurement of the accuracy of the outcome, as well as the best fit. Constrained nonlinear, Levenberg-Marquardt and GaussNewton algorithm are using to solve nonlinear least-square problems. These methods are considered and more appropriate results for calibration of a DC motor are achieved.

\subsection{Constrained Nonlinear Optimization technique}

This technique demonstrates fitting a parameterized simulation using the Constrained Nonlinear Optimization. A generic nonlinear optimization difficulty together with nonlinear parity limitation and nonlinear impurity restrictions can be settled by using a continuous quadratic programming technique. Quadratic programming is a particular type of nonlinear programming. When an objective function is second-class and restrictions are linear, quadratic programming methods are applied.

In Fig. 2, the reference is an objective and constraint function that implements the nonlinear function to minimize. The connections to the Constrained Nonlinear Optimization function are shown in Fig. 2.

- A reference is an objective and constraint function that implements the nonlinear function to minimize.

- Bounds is a group which includes the upper and lower numerical restrictions for parameters which 
objective and constraint function

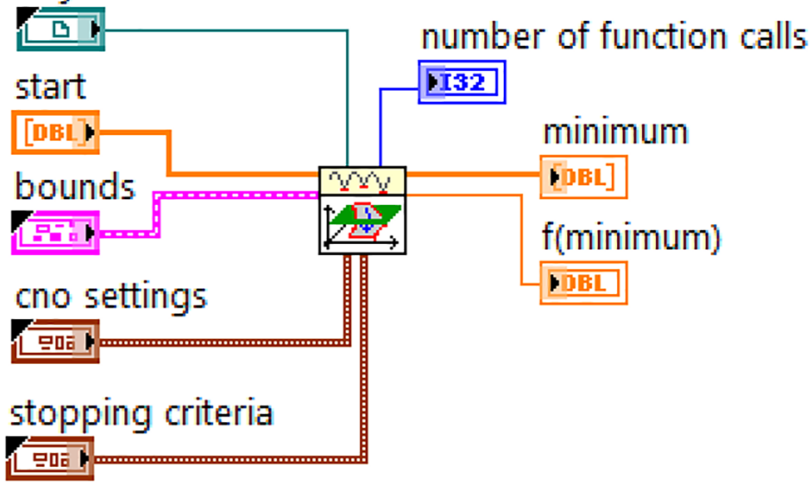

Fig. 2 Constrained Nonlinear Optimization technique

become optimum and the imparity limitations. Least value has the least permissive value of the parameters which become optimum. Maximum value contains the highest allowed value of the parameters being optimized.

- Function calls are equal to objective function calls in the optimization procedure.

- Local minimum in $\mathrm{n}$ dimension is specified by minimum.

- $f$ (minimum) is the value of objective function at the specified minimum.

The cost function with evaluate Position and Velocity and Current is calculated. Also, no equality or inequality constraints are assumed for this problem.

\subsection{Gauss-Newton Optimization technique}

The purpose of Subsection 3.2 is to parameter estimate a user-defined model. A linear or nonlinear model can be defined and then the System Identification Estimate UserDefined Model can be used to estimate this defined model.

Subsection 3.2 summarizes the algorithms of the GaussNewton Optimization technique. The Gauss-Newton Optimization method can be applied to refine the estimation of continuous transfer function models, user-defined models, partially known models, and polynomial models such as the ARMAX, Output-Error (OE), Box-Jenkins (BJ), and General-Linear (GL) models.

The connections to the Gauss-Newton Optimization function are shown in Fig. 3.

A user-defined model estimated by refining the initial estimates using Gauss-Newton Optimization routines.

The purpose of Gauss-Newton Optimization is to minimize the cost function $\left(V_{N}(\theta)\right)$. Also, the purpose of model estimation is to identify the model coefficients

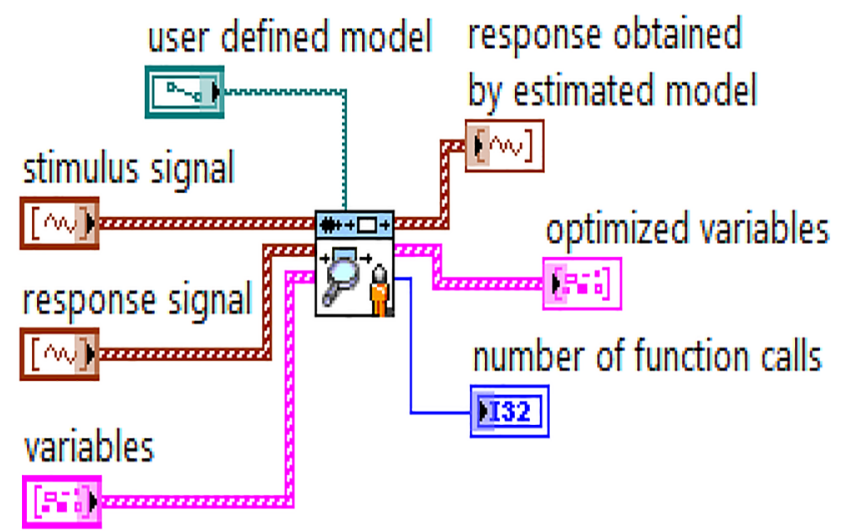

Fig. 3 Gauss-Newton Optimization technique

by minimizing the mean square error $\left(V_{N}(\theta)\right)$, which is defined by the following equations (Eqs. (10) and (11)):

$V_{N}(\theta)=\frac{1}{N} \sum_{k=1}^{N} \frac{1}{2} e^{2}(k, \theta)$,

$e(k, \theta)=\hat{y}(k, \theta)-y(k)$,

where $\theta$ is the variable vector, $\hat{y}(k, \theta)$ is the response to the user-defined model calculates, and $y(k)$ is the measured response. In this equation, all the coefficients to be estimated are combined together as a vector $\theta$. The $e(k, \theta)$ is the error, indicating the difference between the predicted or simulated output of the system $(\hat{y}(k, \theta))$, and the measured output $(y(k))$.

Some methods, such as the multi-stage method for polynomial models, can be used to get a coarse estimation of $\theta$. The following iteration can be assumed to refine $\theta$ :

$\theta^{(i+1)}=\theta^{(i)}+a f\left(\theta^{(i)}\right)$,

where $a$ is the step size and $f\left(\theta^{(i)}\right)$ is the search direction. The purpose of iteration is to minimize the mean square error $V_{N}(\theta)$. The problem to solve lies in how to select and compute the search direction.

The Algorithm Gauss-Newton minimization defines the search direction $f(\theta)$ as:

$$
\begin{aligned}
& f(\theta)=-\left[V^{\prime \prime}(\theta)\right]^{-1}, V^{\prime}(\theta), \\
& V^{\prime}(\theta)=-\frac{1}{N} \sum_{k=1}^{N} \psi(k, \theta), e(k, \theta), \\
& V^{\prime \prime}(\theta) \approx \frac{1}{N} \sum_{k=1}^{N} \psi(k, \theta), \psi^{T}(k, \theta), \\
& \psi(k, \theta)=\frac{d y(k, \theta)}{d k} .
\end{aligned}
$$


$T$ denotes transposing. By inserting Eqs. (14) and (15) into Eq. (13), we obtain the following equation:

$$
\begin{aligned}
& f(\theta)=\frac{\sum_{k=1}^{N} \psi(k, \theta) e(k, \theta)}{\sum_{k=1}^{N} \psi(k, \theta) \psi^{T}(k, \theta)} \\
& =\frac{\left[\begin{array}{llll}
\psi(1, \theta) & \psi(2, \theta) & \ldots & \psi(N, \theta)
\end{array}\right]\left[\begin{array}{c}
e(1, \theta) \\
e(2, \theta) \\
\ldots \\
e(N, \theta)
\end{array}\right]}{\left[\begin{array}{llll}
\psi(1, \theta) & \psi(2, \theta) & \ldots & \psi(N, \theta)
\end{array}\right]\left[\begin{array}{c}
\psi^{T}(1, \theta) \\
\psi^{T}(2, \theta) \\
\ldots \\
\psi^{T}(N, \theta)
\end{array}\right]}
\end{aligned}
$$

$$
=\left[\begin{array}{c}
\psi^{T}(1, \theta) \\
\psi^{T}(2, \theta) \\
\ldots \\
\psi^{T}(N, \theta)
\end{array}\right]^{-1}\left[\begin{array}{c}
e(1, \theta) \\
e(2, \theta) \\
\ldots \\
e(N, \theta)
\end{array}\right] .
$$

$f(\theta)$ can evaluated by solving the following linear equation:

$$
\left[\begin{array}{c}
\psi^{T}(1, \theta) \\
\psi^{T}(2, \theta) \\
\cdots \\
\psi^{T}(N, \theta)
\end{array}\right] f(\theta)=\left[\begin{array}{c}
e(1, \theta) \\
e(2, \theta) \\
\cdots \\
e(N, \theta)
\end{array}\right] .
$$

The following equations define $\psi(k, \theta)$ to calculate the gradients

$\psi(k, \theta)=\frac{y(k, \theta+d \theta)-y(k, \theta-d \theta)}{2, d \theta}$

$d \theta=\theta \cdot$ DIFF_2,

where: $D I F F_{\_} 2=3.2057501263 E-6$.

\subsection{Nonlinear Levenberg-Marquardt Fit Theory}

The nonlinear Lev-Mar notion is applied in order to compute the best-fit parameters which minimize the weighted mean square error among the observations in $Y$ and the best nonlinear fit.

The Nonlinear Lev-Mar Fit Theory specifies the group of coefficients $\left(a_{1}, a_{2}, \ldots, a_{M}\right)$ which fits the observations. The best-fit factors minimize the below equation (chisquare quantity), which characterizes the interval among the curve and the fitted model:

$\sum_{i=0}^{N-1} w_{i}\left(y_{i}-f\left(x_{i} ; a_{1}, a_{2}, \ldots, a_{M}\right)\right)^{2}$, where $N$ is the longitude of $Y$ and $y_{i}, x_{i}$ and $w_{i}$ are the $i$-th element of $Y, X$ and Weight, respectively. Where $X$ is the autonomous variable and $A=\left\{a_{1}, a_{2}, \ldots, a_{M}\right\}$ are undefined coefficients.

In this equation, $\left(x_{i}, y_{i}\right)$ are the input, and $f\left(x_{i} ; a_{1}, a_{2}, \ldots, a_{M}\right)=f(X, A)$ is the nonlinear. If the measurement errors are absolute and normally divided with fixed standard deviation, this is also the least-square approximation.

However, when there is a nonlinear relation, the Nonlinear Lev-Mar Fit function can be used to determine the coefficients. The Lev-Mar method can be used, which is very strong, due to find the factors $A$ of the nonlinear relation among $A$ and $y[i]$.

As an initial step, it needed to determine the nonlinear function $y=f(X, A)$ where the group of factors $(A)$, is defined by the Lev-Mar algorithm.

The correlations to the Constrained Nonlinear Lev-Mar Fit technique are declared below in Fig. 4.

- Inputs are $X$ and $Y$.

- First supposal parameters are our primary guess as to what the factor values are. Using the Nonlinear Lev-Mar.

- Best fit factors related to the values of coefficients $A$ which fit the model of empirical data.

In calculation, the Lev-Mar technique, is well-known as the damped least square method, and it is applied in order to dissolve nonlinear least-square difficulties. These minimization subjects appeared mostly in least-square curve fitting.

The following equation defines the curve model:

$y[i]=f\left(x[i], a_{0}, a_{1}, a_{2}, \ldots\right)$.

The purpose of curve fitting is to find a nonlinear function $f(X, A)$ for the data $\left(x_{i}, y_{i}\right)$ where $i=0,1,2, \ldots, n-1$.

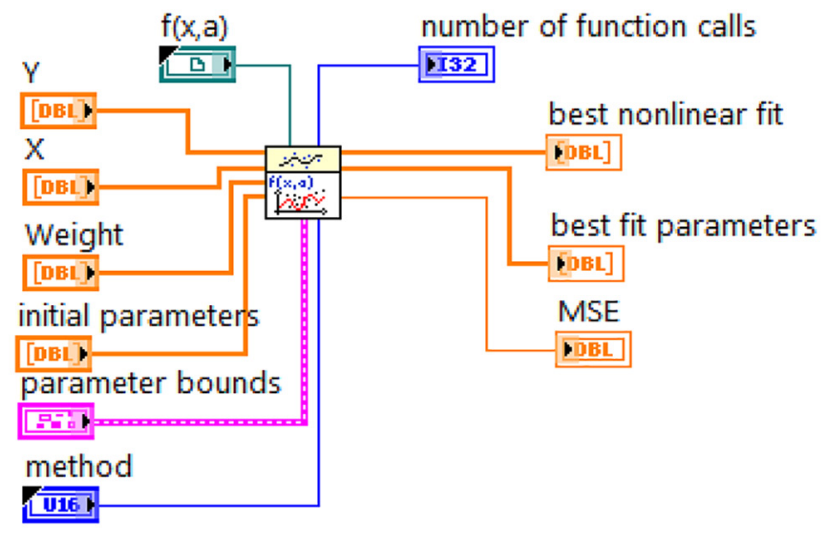

Fig. 4 Nonlinear Lev-Mar Fit 
The function $f(X, A)$ minimizes the residual (MSE) under the weight $W$. The residual is the distance between the data samples and $f(X, A)$. A smaller residual (MSE) means a better fit. In geometry, curve fitting is a curve $Y=f(X, A)$ that fits the data $\left(x_{i}, y_{i}\right)$. In nonlinear curve fitting, supposed that there is previously aware of the nonlinear relations among the absolute parameter $x$ and dependent parameter $y$.

The Lev-Mar algorithm receives $X$ and the group of factors $\left(a_{1}, a_{2}, \ldots, a_{M}\right)$ as inputs and evokes $f\left(X ; a_{1}, a_{2}, \ldots, a_{M}\right)$ and a $2 \mathrm{D}$ array of the partial derivatives regards to the factors. The below equations describes the layout of the sectional derivative matrix of a function with $M$ coefficients and $n X$ values:

$$
f^{\prime}(X, A)=\left[\begin{array}{cccc}
\frac{\partial f\left(x_{0}, A\right)}{\partial a_{1}} & \frac{\partial f\left(x_{0}, A\right)}{\partial a_{2}} & \ldots & \frac{\partial f\left(x_{0}, A\right)}{\partial a_{M}} \\
\frac{\partial f\left(x_{1}, A\right)}{\partial a_{1}} & \frac{\partial f\left(x_{1}, A\right)}{\partial a_{2}} & \ldots & \frac{\partial f\left(x_{1}, A\right)}{\partial a_{M}} \\
\vdots & \vdots & \vdots & \vdots \\
\frac{\partial f\left(x_{n-1}, A\right)}{\partial a_{1}} & \frac{\partial f\left(x_{n-1}, A\right)}{\partial a_{2}} & \ldots & \frac{\partial f\left(x_{n-1}, A\right)}{\partial a_{M}}
\end{array}\right] .
$$

The number of columns $(M)$ in the matrix is equivalent to the number of passive coefficients, while the number of rows in the matrix is equivalent to the number of $X$ values.

\section{Model}

The comparison platform for calibration of DC motor by using nonlinear optimization techniques are implemented by LabVIEW with the control design and simulation module. LabVIEW is an optical programing language and, is a platform for designing systems and extension environment that was targeted at enabling the whole formations of the system to be developed. It is used most by companies that manufacture hardware of some sort, specifically in the groups responsible for testing that the product was designed correctly and that the product works before leaving the factory. It's also considered in researches (i.e. government labs and universities) that use LabVIEW to facilitate their experiments. Also, LabVIEW is used across many industry verticals within the manufacturing realm, including: Military aerospace, Automotive, Semiconductor.

The initial platform consists of two tabs: Parameters range and Identification. The parameters range tab is showing the initial parameters for the simulation. In addition, there is an excitation profile which is the Fourier transform of the time-related pulse form and defines the width, uniformity, and phase of the frequency spectrum excited.

In identification tab, there are three methods which are going to be compared. In Table 2, there are parameters which start with the initial value that we set in the parameters tab, before. When the simulation runs the parameters start with the initial condition. For each parameter the identify button must be clicked to start calibration of the DC motor. During this, parameters automatically are tuning when it achieves to a stable range. Then, the parameters can be accepted and the calibration is done.

\section{Result and discussion}

In Section 5, three nonlinear optimization techniques for calibration of a DC motor are going to compare. The main parameters which are investigated in this problem are: Position, Velocity and Current. In Table 1, there are some real world parameters with desired values. In Results, the main parameters are tried to control such as: Position, Velocity and Current, and the parameters which are defined in Table 1.

\subsection{Result Levenberg-Marquardt method}

\subsubsection{Lev-Mar (least square)}

By using initial conditions, the program for this method is running.

As can be seen from the Fig. 5, the calibrated response is tracking the simulated response in three concepts: position, velocity and current. In the plots, it was illustrated that the measured response is far from both simulated and calibrated response.

Table 2, is the results of parameters and Errors for each parameter. Parameters are almost close to initial conditions in comparison to the real world values.

\subsubsection{Lev-Mar (bi-square)}

Here, the results are shown as Fig. 6. As can be seen the calibration response for position and velocity perform very

Table 2 Parameters after run in Lev-Mar (least square) method

\begin{tabular}{lcc}
\hline Parameters & Identified Parameters & Error \\
\hline$R(\mathrm{Ohm})$ & 4.98834 & 1.98834 \\
$L(\mathrm{Henry})$ & 0.499045 & 0.09900451 \\
$K_{e}(\mathrm{~N} \mathrm{~m} / \mathrm{A})$ & 0.00234385 & -0.00015615 \\
$K_{t}(\mathrm{~N} \mathrm{~m} / \mathrm{A})$ & 0.00258264 & $8.26434 \mathrm{E}-5$ \\
$J\left(\mathrm{Kg} \mathrm{m} / \mathrm{s}^{2}\right)$ & 0.00495403 & $-4.59719 \mathrm{E}-5$ \\
$B(\mathrm{~T})$ & 0.0996606 & -0.00033971 \\
\hline
\end{tabular}




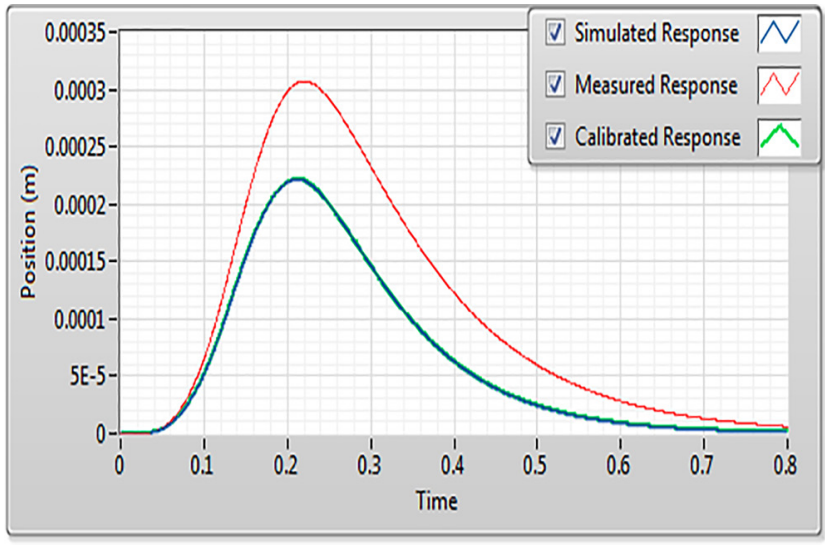

(a)

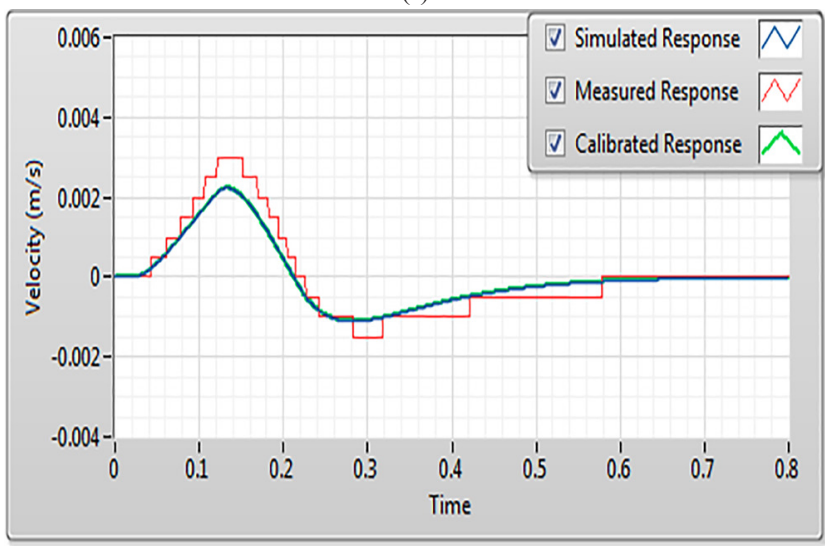

(b)

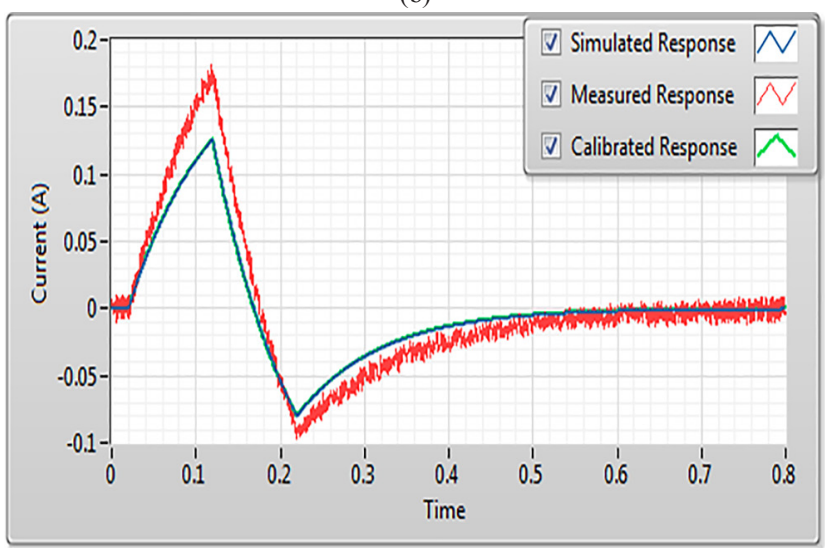

(c)

Fig. 5 Result Lev-Mar (least square) method; (a) Results for the position; (b) Results for the velocity; (c) Results for the current.

well and track the simulation response effectively. But, in current plot, there are more disturbances and chaoses.

Table 3, shows the parameters after stopping the program.

\subsubsection{Lev-Mar (least absolute residual)}

In this method, the results are as Fig. 7. As it declared the calibration response has overlap with simulation response and measured response. Table 4, shows parameters after

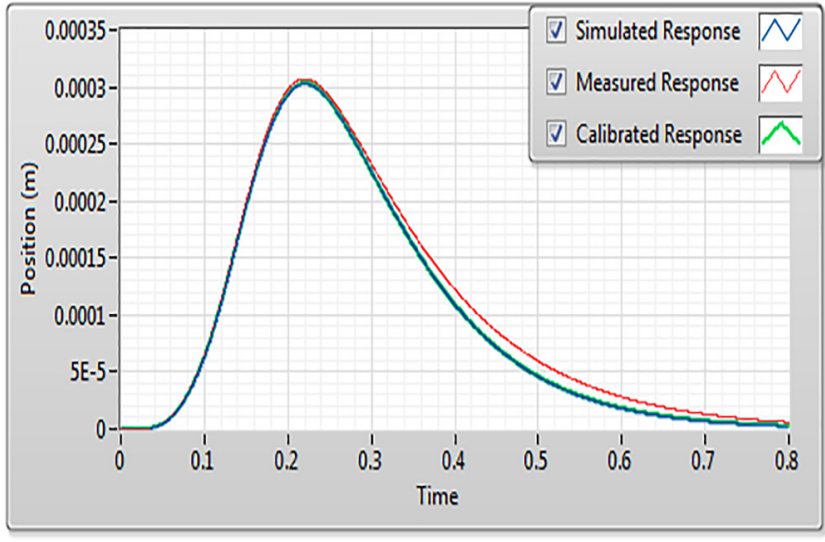

(a)

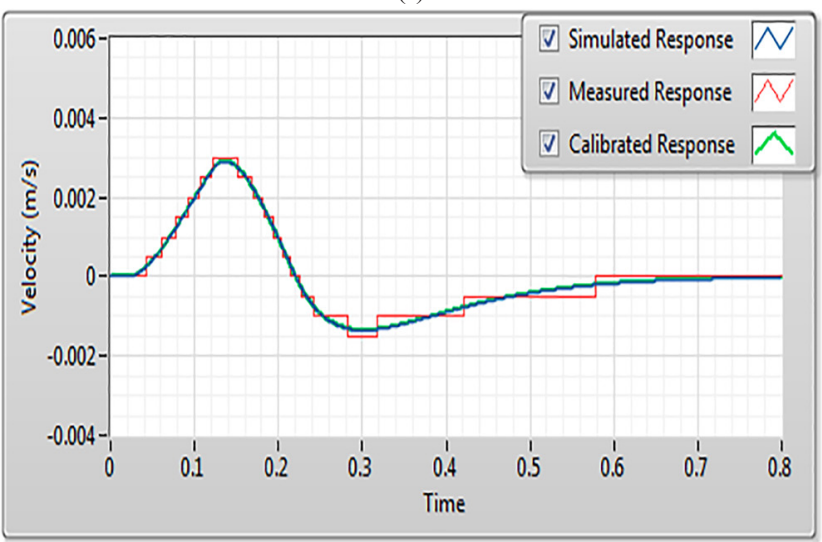

(b)

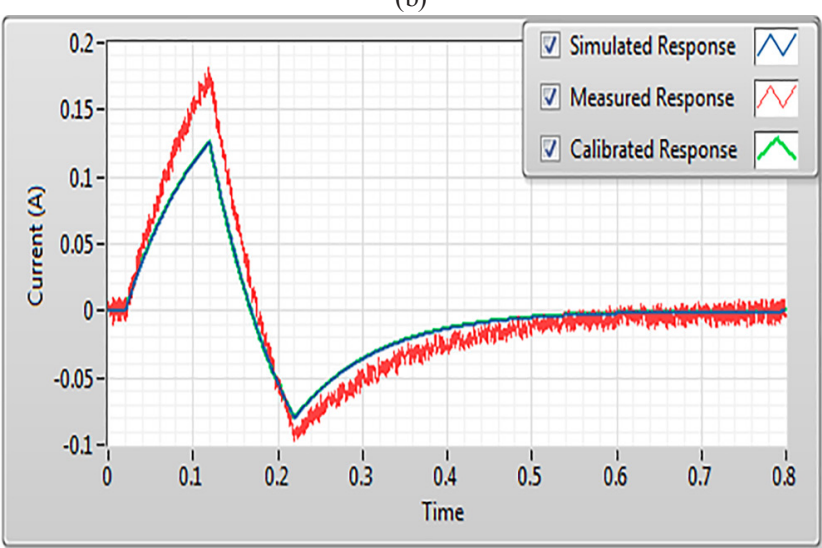

(c)

Fig. 6 Result Lev-Mar (bi-square) method; (a) Results for the position; (b) Results for the velocity; (c) Results for the current.

Table 3 Parameters after run in Lev-Mar (bi-square) method

\begin{tabular}{lcc}
\hline Parameters & Identified Parameters & Error \\
\hline$R(\mathrm{Ohm})$ & 4.99999 & 1.99999 \\
$L($ Henry $)$ & 0.500114 & 0.100114 \\
$K_{e}(\mathrm{~N} \mathrm{~m} / \mathrm{A})$ & 0.0024995 & $-4.98257 \mathrm{E}-7$ \\
$K_{t}(\mathrm{~N} \mathrm{~m} / \mathrm{A})$ & 0.00383366 & 0.00133366 \\
$J\left(\mathrm{Kg} \mathrm{m}^{2} / \mathrm{s}^{2}\right)$ & 0.00657493 & 0.00157493 \\
$B(\mathrm{~T})$ & 0.0998273 & -0.000173012 \\
\hline
\end{tabular}




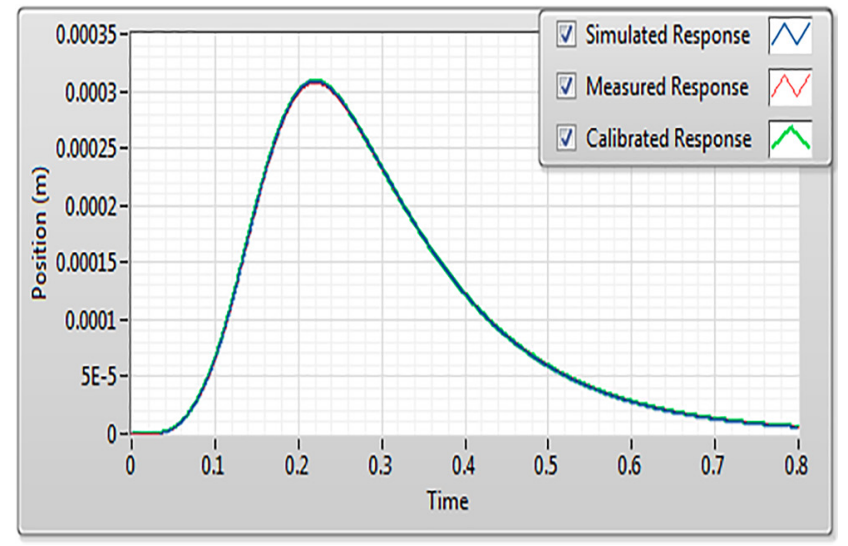

(a)

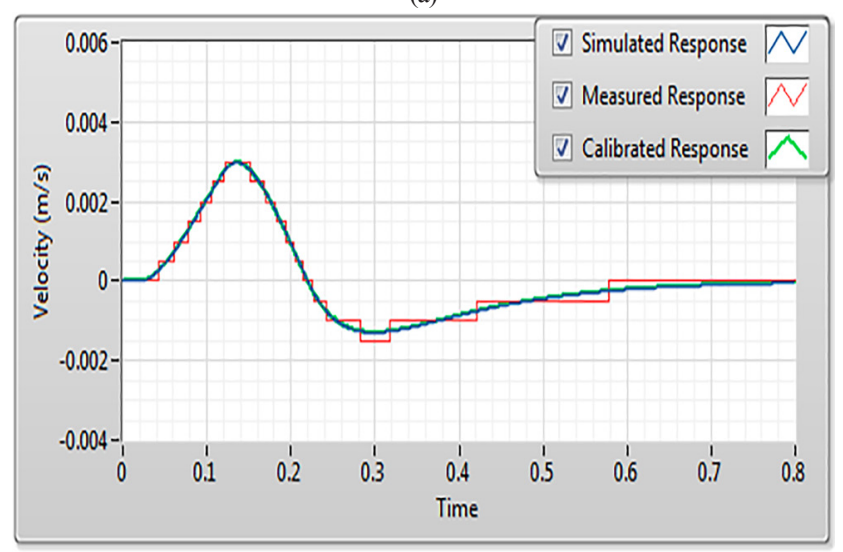

(b)

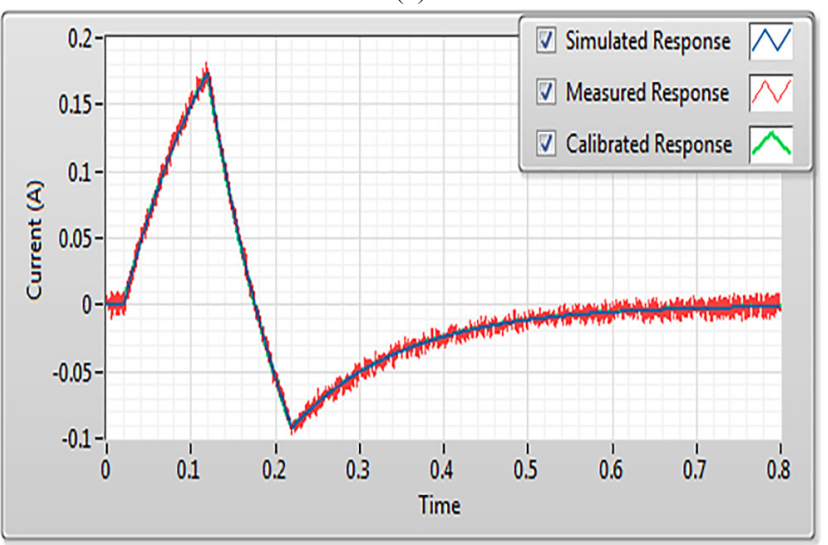

(c)

Fig. 7 Result Lev-Mar (least absolute residual) method; (a) Results for the position; (b) Results for the velocity; (c) Results for the current.

Table 4 Parameters after run in Lev-Mar (least absolute residual) method

\begin{tabular}{lcc}
\hline Parameters & Identified Parameters & Error \\
\hline$R(\mathrm{Ohm})$ & 3.06112 & 0.0616486 \\
$L($ Henry $)$ & 0.40934 & 0.0094177 \\
$K_{e}(\mathrm{~N} \mathrm{~m} / \mathrm{A})$ & $1 \mathrm{E}-5$ & -0.00249 \\
$K_{t}(\mathrm{~N} \mathrm{~m} / \mathrm{A})$ & 0.00252899 & $2.81609 \mathrm{E}-5$ \\
$J\left(\mathrm{Kg} \mathrm{m} / \mathrm{s}^{2}\right)$ & 0.00487799 & -0.000129496 \\
$B(\mathrm{~T})$ & 0.098828 & -0.00113768 \\
\hline
\end{tabular}

simulation. By setting the simulation method on LevMar (least absolute residual) technique and running the method, parameters can be accepted after identifying.

\subsection{Result Gauss-Newton method}

In Subsection 5.2, the program is switched on GaussNewton method. After this simulation, it can be seen that the calibration response performed very well and it is tracking the simulated response and it also is same as measured response Fig. 8 and Table 5, is the results of parameters after simulation.

\subsection{Result Constrained Nonlinear Optimization method} The last method is constraint nonlinear optimization. As can be seen from the Fig. 9, the calibration response in the concept of position is tracking the simulated response, but in the velocity and current we have a little bit chaos. Table 6, shows the parameters after running the program.

After these results, the results in three concepts are going to compare such as: cost function, number of function calls and MSE. Here, in Table 7, the comparison of methods is shown.

\section{Conclusion}

In this paper, the goal was to estimate the parameters of a DC motor, called calibration, by using three nonlinear optimization techniques and the goal is to make a comparison to identify which method is performing well. These three methods are Lev-Mar, Gauss-Newton, and constraint nonlinear optimization. First of all, initial parameters as well as in the real world are defined, then the program applied for each method.

After running, by clicking the identify button the program start to tuning the parameters and calibrated them to achieve the simulation response. After a number of function calls the program staying stable and parameters can be accepted. The results show that the Lev-Mar methods perform very well with lower MSE. It means the calibration response has overlap with the simulation response. Here MSE is important for us because it shows that the method performs well with a low number of errors. However, like several fitting methods, the Lev-Mar detects just local minimums, which are not as a matter of course, the global minimums.

The Lev-Mar inserts among the Gauss-Newton Algorithm (GNA) and the technique of gradient descent. Lev-Mar is stronger than the Gauss-Newton, which in several cases it detects a solution even while it begins very 


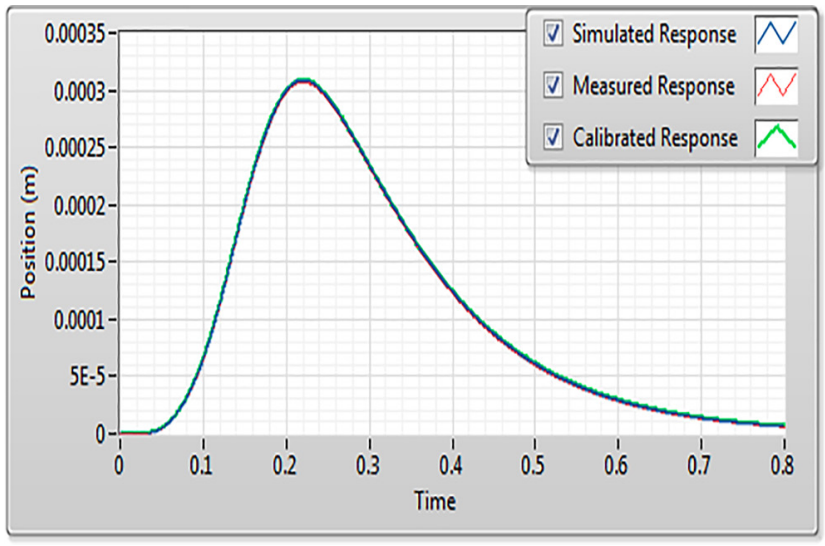

(a)

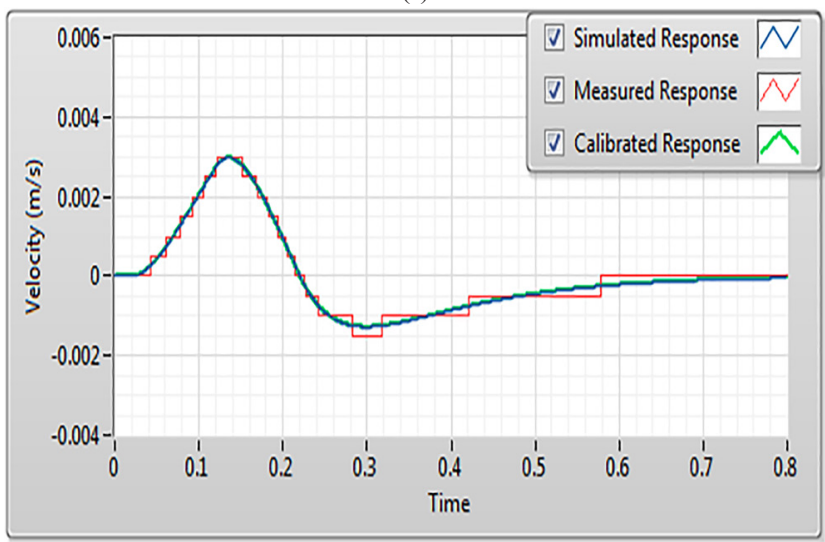

(b)

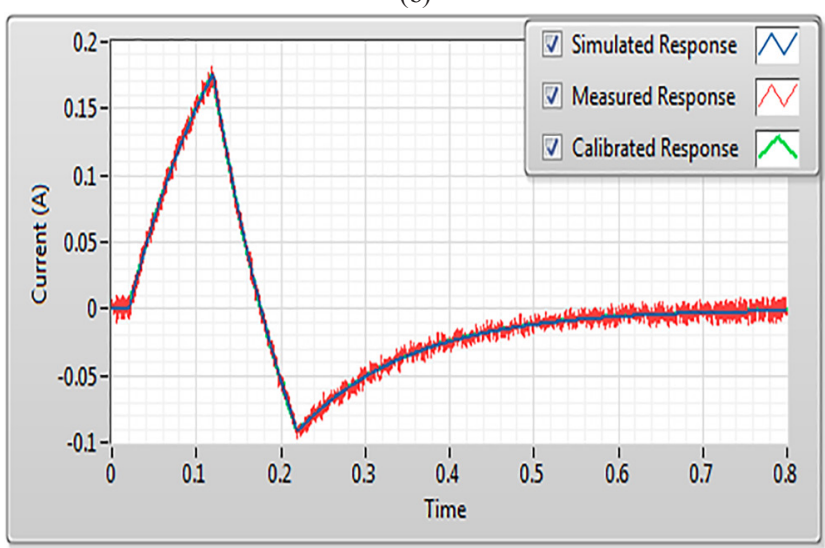

(c)

Fig. 8 Result Gauss-Newton method; (a) Results for the position; (b) Results for the velocity; (c) Results for the current.

Table 5 Parameters after run in Gauss-Newton method

\begin{tabular}{lcc}
\hline Parameters & Identified Parameters & Error \\
\hline$R(\mathrm{Ohm})$ & 2.95541 & -0.0445906 \\
$L($ Henry $)$ & 0.403294 & 0.00329365 \\
$K_{e}(\mathrm{~N} \mathrm{~m} / \mathrm{A})$ & 0.01 & 0.0075 \\
$K_{t}(\mathrm{~N} \mathrm{~m} / \mathrm{A})$ & 0.00252173 & $2.17299 \mathrm{E}-5$ \\
$J\left(\mathrm{Kg} \mathrm{m}^{2} / \mathrm{s}^{2}\right)$ & 0.00496237 & $-3.76258 \mathrm{E}-5$ \\
$B(\mathrm{~T})$ & 0.100691 & 0.000691178 \\
\hline
\end{tabular}

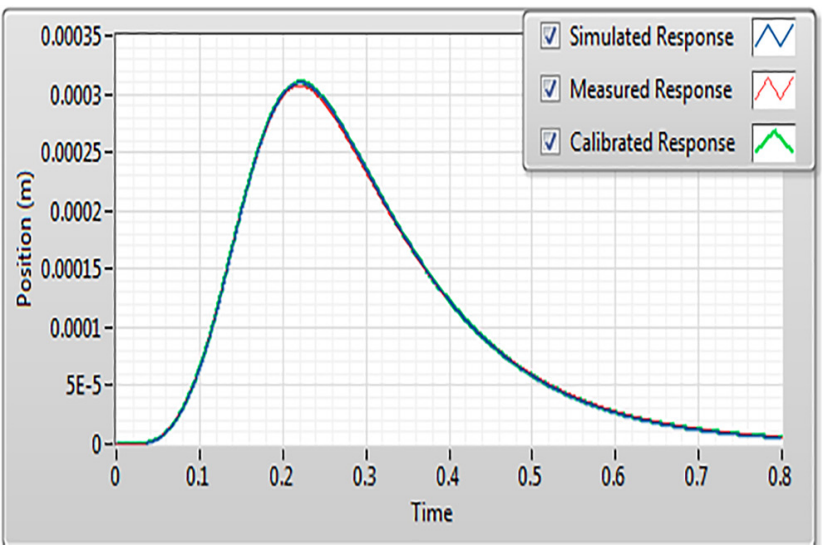

(a)

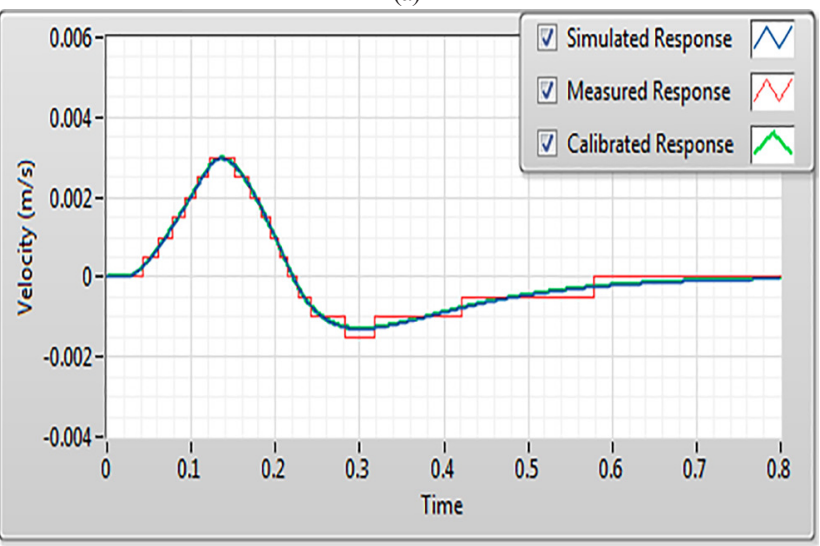

(b)

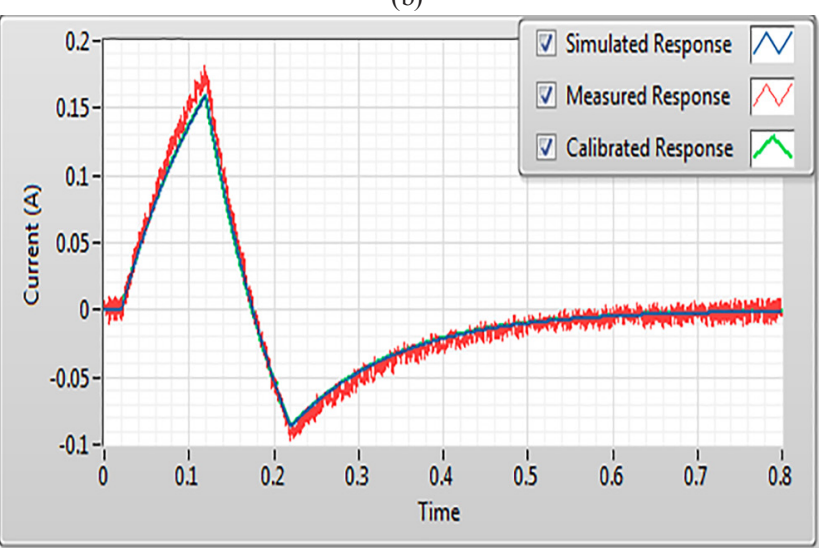

(c)

Fig. 9 Result Constrained Nonlinear Optimization method; (a) Results for the position; (b) Results for the velocity; (c) Results for the current.

Table 6 Parameters after run in Constrained Nonlinear Optimization method

\begin{tabular}{lcc}
\hline Parameters & Identified Parameters & Error \\
\hline$R(\mathrm{Ohm})$ & 3.03953 & 0.0395296 \\
$L($ Henry $)$ & 0.397634 & -0.00236579 \\
$K_{e}(\mathrm{~N} \mathrm{~m} / \mathrm{A})$ & 0.0024586 & $-4.13982 \mathrm{E}-5$ \\
$K_{t}(\mathrm{~N} \mathrm{~m} / \mathrm{A})$ & 0.00361373 & 0.00111373 \\
$J\left(\mathrm{Kg} \mathrm{m}^{2} / \mathrm{s}^{2}\right)$ & 0.00735488 & 0.00235488 \\
$B(\mathrm{~T})$ & 0.143119 & 0.043119 \\
\hline
\end{tabular}


Table 7 Comparison of mentioned methods

\begin{tabular}{|c|c|c|c|}
\hline \multirow[b]{2}{*}{ Methods } & \multicolumn{3}{|c|}{ Parameters } \\
\hline & $\begin{array}{c}\text { Cost } \\
\text { Function }\end{array}$ & $\begin{array}{l}\text { function } \\
\text { calls }\end{array}$ & MSE \\
\hline Lev-Mar (least square) & 2.08801 & 91 & $8.82137 \mathrm{E}-5$ \\
\hline Lev-Mar (bi-square) & 2.10319 & 494 & $1.57821 \mathrm{E}-12$ \\
\hline $\begin{array}{l}\text { Lev-Mar (least-absolute- } \\
\text { residual) }\end{array}$ & 0.0748517 & 325 & 0.00136936 \\
\hline Gauss-Newton & 0.0561348 & 67 & 0.0013293 \\
\hline $\begin{array}{l}\text { Constrained Nonlinear } \\
\text { Optimization }\end{array}$ & 0.0885244 & 275 & 0.00111373 \\
\hline
\end{tabular}

\section{References}

[1] Chen, D., Yu, Y., Xu, L., Liu, X. "Kalman Filtering for Discrete Stochastic Systems with Multiplicative Noises and Random TwoStep Sensor Delays", Discrete Dynamics in Nature and Society, 2015, Article ID: 809734, 2015.

https://doi.org/10.1155/2015/809734

[2] Cherifi, D., Miloud, Y. "Improved Sensorless Control of Doubly Fed Induction Motor Drive Based on Full Order Extended Kalman Filter Observer", Periodica Polytechnica Electrical Engineering and Computer Science, 64(1), pp. 64-73, 2020. https://doi.org/10.3311/PPee.14245

[3] Ruderman, M., Krettek, J., Hoffmann, F., Bertram, T. "Optimal State Space Control of DC Motor", IFAC Proceedings Volumes, 41(2), pp. 5796-5801, 2008.

https://doi.org/10.3182/20080706-5-KR-1001.00977

[4] Chevrel, P., Sicot, L., Siala, S. "Switched LQ controllers for DC motor speed and current control: a comparison with cascade control", In: PESC Record. 27th Annual IEEE Power Electronics Specialists Conference, Baveno, Italy, 1996, pp. 906-912. https://doi.org/10.1109/PESC.1996.548689

[5] Liu, A., Liang, Y., Gao, S., Gao, J. "Modified linear quadratic optimal control method and application in linear brushless direct current motor", In: 2007 International Conference on Electrical Machines and Systems (ICEMS), Seoul, South Korea, 2007, pp. 1829-1834. [online[ Available at: https://ieeexplore.ieee.org/ abstract/document/4412105 [Accessed: 25 May 2020]

[6] Dezaki, H., Abyaneh, H., Agheli, A., Mazlumi, K. "Optimized Switch Allocation to Improve the Restoration Energy in Distribution Systems", Journal of Electrical Engineering, 63(1), pp. 47-52, 2012.

https://doi.org/10.2478/v10187-012-0007-9

[7] Orosz, T., Sleisz, Á., Tamus, Z. Á. "Metaheuristic Optimization Preliminary Design Process of Core-Form Autotransformers", IEEE Transactions on Magnetics, 52(4), Article Number: 8400310, 2016. https://doi.org/10.1109/TMAG.2015.2496905

[8] Orosz, T. "Evolution and Modern Approaches of the Power Transformer Cost Optimization Methods", Periodica Polytechnica Electrical Engineering and Computer Science, 63(1), pp. 37-50, 2019. https://doi.org/10.3311/PPee.13000 out of the final minimum. Lev-Mar has an inclination to be slower than Gauss-Newton for well-performed functions and sensible starting parameters. Also, Lev-Mar can be Gauss-Newton by using a trust-region method.

[9] Pánek, D., Orosz, T., Karban, P. "Artap: Robust Design Optimization Framework for Engineering Applications", In: 2019 Third International Conference on Intelligent Computing in Data Sciences (ICDS), Marrakech, Morocco, 2019, pp. 1-6, 2019. https://doi.org/10.1109/ICDS47004.2019.8942318

[10] Maanani, Y., Menacer, A. "Modeling and Diagnosis of the Inter-Turn Short Circuit Fault for the Sensorless Input-Output Linearization Control of the PMSM", Periodica Polytechnica Electrical Engineering and Computer Science, 63(3), pp. 159-168, 2019. https://doi.org/10.3311/PPee.13658

[11] Vajsz, T., Számel, L. "Improved Modified DTC-SVM Methods for Increasing the Overload-capability of Permanent Magnet Synchronous Motor Servo- and Robot Drives - Part 1", Periodica Polytechnica Electrical Engineering and Computer Science, 62(3), pp. 65-73, 2018. https://doi.org/10.3311/PPee.11744

[12] Vajsz, T., Számel, L. "Improved Modified DTC-SVM Methods for Increasing the Overload-capability of Permanent Magnet Synchronous Motor Servo- and Robot Drives - Part 2", Periodica Polytechnica Electrical Engineering and Computer Science, 62(3), pp. 74-81, 2018. https://doi.org/10.3311/PPee.11762

[13] Honório, L. M., Barros Costa, E., Oliveira, E. J., de Almeida Fernandes, D., Moreira, A. P. G. M. "Persistently-exciting signal generation for Optimal Parameter Estimation of constrained nonlinear dynamical systems", ISA Transactions, 77, pp. 231-241, 2018. https://doi.org/10.1016/j.isatra.2018.03.024

[14] Roy, A., Srivastava, S. "Design of optimal PI $\lambda \mathrm{D} \delta$ controller for speed control of DC motor using constrained particle swarm optimization", In: 2016 International Conference on Circuit, Power and Computing Technologies (ICCPCT), Nagercoil, India, 2016, pp. 1-6. https://doi.org/10.1109/ICCPCT.2016.7530150

[15] Khelifa, M., Mordjaoui, M., Medoued, A. "An inverse problem methodology for design and optimization of an interior permanent magnetic BLDC motor", International Journal of Hydrogen Energy, 42(28), pp. 17733-17740, 2017. https://doi.org/10.1016/j.ijhydene.2017.02.017 
[16] Sundareswaran, K., Bhattacharjee, A. "A Novel Stochastic Optimization Algorithm Inspired From The Biology Of Plant Reproduction", In: 2019 IEEE International Conference on Electrical, Computer and Communication Technologies (ICECCT), Coimbatore, India, 2019, pp. 1-6. https://doi.org/10.1109/ICECCT.2019.8869007

[17] Iqbal, T. Amjadullah, Zeb, K. "Performance of grid interfaced doubly fed induction generator-wind turbine using fuzzy logic controller based on Gauss Newton algorithm under symmetrical and asymmetrical faults", In: 2017 International Conference on Electrical Engineering (ICEE), Lahore, Pakistan, 2017, pp. 1-6. https://doi.org/10.1109/ICEE.2017.7893439

[18] Jansch-Porto, J. P., Hu, B., Dullerud, G. "Convergence Guarantees of Policy Optimization Methods for Markovian Jump Linear Systems", [math.OC], arXiv:2002.04090, Cornell University, Ithaca, NY, USA, 2020. [online] Available at: https://arxiv.org/ abs/2002.04090 [Accessed: 01 June 2020]

[19] Putra, S., Mu'tamar, K., Zulkarnain "Estimation of Parameters in the SIR Epidemic Model Using Particle Swarm Optimization", American Journal of Mathematical and Computer Modelling, 4(4), pp. 83-93, 2019.

https://doi.org/10.11648/j.ajmcm.20190404.11

[20] Li, J., Zheng, W. X., Gu, J., Hua, L. "Parameter estimation algorithms for Hammerstein output error systems using LevenbergMarquardt optimization method with varying interval measurements", Journal of the Franklin Institute, 354(1), pp. 316-331, 2017. https://doi.org/10.1016/j.jfranklin.2016.10.002
[21] Li, Y., Wang, X., Lu, S. "Fractional Order Parameter Identification Of PMSM Based on Improved Levenberg-Marquardt Algorithm", In: 2019 Chinese Automation Congress (CAC), Hangzhou, China, 2019, pp. 4219-4223. https://doi.org/10.1109/CAC48633.2019.8996888

[22] Mellah, H., Hemsas, K. E., Taleb, R. "Intelligent Sensor Based Bayesian Neural Network for Combined Parameters and States Estimation of a Brushed DC Motor", International Journal of Advanced Computer Science and Applications (IJACSA), 7(7), pp. 230-235, 2016.

https://doi.org/10.14569/IJACSA.2016.070731

[23] Zeb, K., Uddin, W., Haider, A., Belal, S., Mehmood, C. A., Khan, M. A., Kim, H. J. "Robust speed regulation of indirect vector control induction motor using fuzzy logic controllers based on optimization algorithms", Electrical Engineering, 100(2), pp. 787-802, 2018. https://doi.org/10.1007/s00202-017-0553-Z

[24] Alhanjouri, M. A. "Speed Control of DC Motor Using Artificial Neural Network", International Journal of Science and Research (IJSR), 7(3), pp. 2140-2148, 2017. [online] Available at: http://hdl. handle.net/20.500.12358/24633 [Accessed: 01 June 2020] 